\title{
Dimensional analysis
}

Geometry has surprising consequences for the behaviour of matter. Living in three dimensions, we're familiar with liquids that abruptly freeze into solids, or crystals under pressure that suddenly alter their molecular structures. Confine the same materials within a narrow, roughly one-dimensional (1D) wire, and everything changes. In one dimension, molecular interactions can't overcome the disrupting influence of noise to create long-range order; liquids won't freeze at any temperature.

We know this from the theory of critical phenomena, which also reveals why geometry is so important - in effect, it controls the crucial supply lines for the forces of order in their battle against disrupting noise. In one dimension, one end of a chain can influence the other end only by interactions transmitted directly along the chain, so any disruption is necessarily 'in the way' and destroys the linking of behaviour in distant parts. In two or more dimensions, multiple paths connect any two points, and the number of possible paths grows rapidly with increasing dimension. Order then emerges out of chaos more readily.

Clearly, all this has more to do with simple geometry than physics, and unsurprisingly its implications are evident elsewhere. A good example arises in evolutionary theory, especially in the effort to extend classical population genetics beyond the simplifying assumptions of early theorists such as Motoo Kimura or Ronald Fisher. They were mostly limited to studying evolution in 'well mixed' populations, in which each individual interacts in equal likelihood with any other, such as bacteria interacting in a well-stirred liquid.

This assumption makes the maths easier, but is rarely, if ever, true in reality. Organisms often don't move around enough to interact with more than a small fraction of others that live nearby. More generally, evolution itself, or the environment, frequently stirs up spatial structure by sorting genetic types preferentially into some regions, thereby strongly skewing subsequent interactions away from the well-mixed ideal.

What does this have to do with dimension? As several researchers have recently noted, such departures from the well-mixed ideal frequently arise in situations in which evolution works in a lower-dimensional setting. In physics, 'well mixed' translates more or less as 'mean field', and mean field theory works well above a certain critical dimension, where noise and fluctuations have

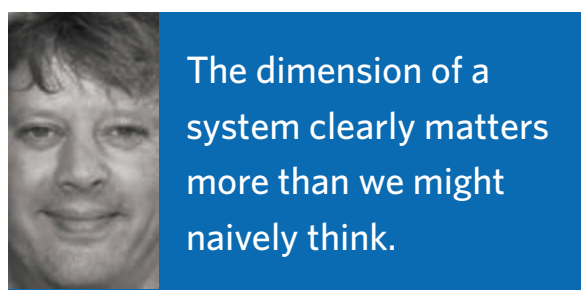

less influence. Below this dimension, we also know, it can be wildly inaccurate.

One of the most common situations, as Kirill Korolev et al. discuss (Rev. Mod. Phys. 82, 1691-1718; 2010), emerges out of the expansion of a population. Say a bacterial colony is expanding by growth into a new food source. In the simplest picture of roughly circular surface growth, individuals at the expanding front exist in a roughly $1 \mathrm{D}$ world; they're almost certainly the offspring of other individuals living in the same front. Experiments show that the boosted power of random fluctuations under such conditions lead to weird effects, including a tendency for strong genetic de-mixing. For example, Oskar Hallatschek et al. studied the spreading of Escherichia coli and Saccharomyces cerevisiae on Petri dishes (Proc. Natl Acad. Sci. USA 104, 19926-19930; 2007). They gave each microbe a (selectively neutral) genetic type - a gene encoding for a protein with one of two distinct fluorescence spectra, so that they could detect bacterial type optically. The interesting and typical outcome is that an initially well-mixed 50/50 population gradually segregates as it grows, so that the front has sharply defined homogeneous domains corresponding to the two types.

This segregation merely reflects an enhanced role for random fluctuations in this low-dimensional system. Biologists have long known that random genetic change (genetic 'drift', as they say) is a powerful effect in evolution. Take a population mixed evenly, $50 \%$ green eyes and $50 \%$ blue, and split off a small subpopulation of 25 individuals: this new group may, by statistical fluctuations alone, be $80 \%$ green-eyed and the imbalance may then persist in the evolution of the new population (hence the term 'founder effect' for the strong loss of genetic diversity associated with the seeding of new populations from small groups).

The same type of effect happens by dimensionality alone for expanding populations. Kirill Korolev and colleagues have studied the phenomenon analytically, using a 'stepping stone' model that breaks the
1D world into small regions, each of which can be considered well-mixed (http://arxiv. org/abs/0904.4625; 2009). Each area is subject to mutation, selection, genetic drift, and there's also migration between neighbouring areas. Their work shows that the spatial segregation of individuals (characterized by two neutral alleles, as in the experiments just described) occurs in two stages. During the first stage, distinguishable domains emerge from the well-mixed population. Then, during a second stage, the boundaries of these regions move randomly and annihilate on collision; some of the domains vanish while others grow.

This is all dramatically different from the well-mixed ideal. One of the interesting consequences, as Korolev et al. point out, is that this spontaneous genetic de-mixing means that natural selection acts only near these domain boundaries, where genetically distinct individuals are present in the same environment. As these boundaries involve only an extremely small fraction of the population, alleles giving evolutionarily disadvantageous traits may well persist in 1D populations long after they would have been wiped out in a well-mixed population. This is in direct analogy to the enhanced persistence of disorder in low-dimensional systems in physics.

These results have been worked out before in the population-genetics literature, but the perspective from physics may be unifying. Dimension clearly matters more than we might naively think, as physicists have learned over the past 25 years, with some 50,000 papers published on low-dimensional systems. Perhaps biology awaits a similar explosion. As in physics, the effect of lower dimension is to disrupt natural ordering influences. This type of effect could also be more widespread, not only in expanding populations - think, for example, of a population living along a river bank, or otherwise confined to some linear structures (grasses, biological tubes in other organisms, or intersecting surfaces).

We're still only beginning to explore these consequences. And it may well be that dimension has influences at the human level as well. As Korolev et al. note, the basic model they've explored has links to the so-called voter model, relevant to the spread of opinions through a human population. It's not a stretch at all to suppose dimensional aspects of basic social interactions. We may just be too close to the action to see it.

\section{MARK BUCHANAN}

